\title{
Chinese expert consensus on the perinatal and neonatal management for the prevention and control of the 2019 novel coronavirus infection (First edition)
}

\author{
Laishuan Wang ${ }^{1 \#}$, Yuan Shi ${ }^{2 \#}$, Tiantian Xiao ${ }^{1,3}$, Jianhua Fu ${ }^{4}$, Xing Feng ${ }^{5}$, Dezhi Mu ${ }^{6}$, Qi Feng \\ Mingyan $\mathrm{Hei}^{8}$, Xiaojing $\mathrm{Hu}^{1}$, Zhankui $\mathrm{Li}^{9}$, Guoping $\mathrm{Lu}^{1}$, Zezhong Tang ${ }^{7}$, Yajuan Wang ${ }^{8}$, \\ Chuanqing Wang ${ }^{1}$, Shiwen Xia ${ }^{10}$, Jianqing Xu ${ }^{11}$, Yujia Yang ${ }^{12}$, Jie Yang ${ }^{13}$, Mei Zeng ${ }^{1}$, Jun Zheng ${ }^{14}$, \\ Wei Zhou $^{15}$, Xiaoyu Zhou ${ }^{16}$, Xiaoguang Zhou ${ }^{16}$, Lizhong Du ${ }^{17}$, Shoo K. Lee ${ }^{18,19}$, Wenhao Zhou ${ }^{1}$; on behalf \\ of the Working Committee on Perinatal and Neonatal Management for the Prevention and Control of \\ the 2019 Novel Coronavirus Infection
}

${ }^{1}$ Department of Pediatrics, Children's Hospital of Fudan University, Shanghai 201102, China; 'Department of Neonatology, Children's Hospital of Chongqing Medical University, Chongqing 401122, China; ${ }^{3}$ Department of Neonatology, Chengdu Women's and Children's Central Hospital, School of Medicine, University of Electronic Science and Technology of China, Chengdu 611731, China; ${ }^{4}$ Department of Neonatology, Shengjing Hospital, China Medical University, Shenyang 110004, China; ${ }^{5}$ Department of Neonatology, Children's Hospital of Soochow University, Suzhou 215025, China; ${ }^{6}$ Department of Pediatrics, West China Second University Hospital, Sichuan University, Chengdu 610041, China; ${ }^{7}$ Department of Pediatrics, Peking University First Hospital, Beijing 100034, China; ${ }^{8}$ Neonatal Center, Beijing Children's Hospital, Capital Medical University, Beijing 100045, China; ${ }^{9}$ Department of Neonatology, Northwest Women and Children's Hospital, Xi'an 610113, China; ${ }^{10}$ Department of Neonatology, Hubei Maternal and Child Health Hospital, Wuhan 430070, China; ${ }^{11}$ Shanghai Public Health Clinical Center \& Institutes of Biomedical Sciences, Fudan University, Shanghai 200000, China; ${ }^{12}$ Department of Pediatrics, Xiangya Hospital, Central South University, Changsha 410008, China; ${ }^{13}$ Department of Neonatology, Guangdong Maternal and Children's Hospital, Guangzhou Medical College, Guangzhou 511400, China; ${ }^{14}$ Department of Neonatology, Tianjin Central Hospital of Gynecology Obstetrics, Tianjin 300052, China; ${ }^{15}$ Department of Neonatology, Guangzhou Women and Children's Medical Center, Guangzhou Medical University Guangzhou 510623, China; ${ }^{16}$ Neonatal Medical Center, Children's Hospital of Nanjing Medical University, Nanjing 210008, China; ${ }^{17}$ Department of Neonatology, the Children's Hospital, Zhejiang University School of Medicine, Hangzhou 310052, China; ${ }^{18}$ Maternal-Infant Care Research Centre and Department of Pediatrics, Mount Sinai Hospital, Toronto, Ontario, Canada; ${ }^{19}$ Department of Pediatrics, Department of Obstetrics and Gynecology and Dalla Lana School of Public Health, University of Toronto, Toronto, Ontario, Canada

\#These authors contributed equally to this work.

Correspondence to: Shoo K. Lee. Neonatologist \& Director, Maternal-Infant Care Research Center (MiCARE), Mount Sinai Hospital; Professor of Pediatrics, Obstetrics \& Gynecology, and Public Health, University of Toronto, Toronto, Ontario, Canada. Email: shoo.lee@sinaihealthsystem.ca; Wenhao Zhou. Department of Neonatology, Children’s Hospital of Fudan University, 399 Wanyuan Road, Shanghai 201102, China. Email: zhouwenhao@fudan.edu.cn.

\begin{abstract}
Since December 2019, there has been an outbreak of novel coronavirus (2019-nCoV) infection in China. Two cases of neonates with positive 2019-nCoV tests have been reported. Due to the immature immune system and the possibility of vertical transmission from mother to infant, neonates have become a high-risk group susceptible to 2019-nCoV, which emphasize a close cooperation from both perinatal and neonatal pediatrics. In neonatal intensive care unit (NICU), to prevent and control infection, there should be practical measures to ensure the optimal management of children potentially to be infected. According to the latest 2019-nCoV national management plan and the actual situation, the Chinese Neonatal 2019-nCoV expert working Group has put forward measures on the prevention and control of neonatal 2019-nCoV infection.
\end{abstract}

Keywords: Novel coronavirus; perinatology; neonate

Submitted Feb 02, 2020. Accepted for publication Feb 06, 2020.

doi: 10.21037/atm.2020.02.20

View this article at: http://dx.doi.org/10.21037/atm.2020.02.20

(c) Annals of Translational Medicine. All rights reserved. 
Since December 2019, the novel coronavirus infection has shown the clinical epidemiological characteristics of rapid spread and the capability to infect a susceptible general population. The pathogen belongs to the $\beta$-type coronavirus (2019 novel coronavirus, 2019-nCoV) (1-3). Its genetic characteristics are different from those of severe acute respiratory syndrome coronavirus (SARS coronavirus, SARS-CoV) and Middle East respiratory distress syndrome coronavirus (MERS coronavirus, MERS-CoV) $(4,5)$. The main route of transmission is via respiratory droplets, and it can also be transmitted through contact. Additionally, current studies have suggested that 2019-nCoV may be transmitted through the fecal-oral route as well (6). Pregnant women have been found infected with $2019-\mathrm{nCoV}$, and the youngest child patient with positive 2019-nCoV test reported thus far is 36 hours old. As of now, two cases of neonates with positive 2019-nCoV tests have been reported and the route of vertical-transmission cannot be ruled out.

In the case that the $2019-\mathrm{nCoV}$ infection does cause potential harm to newborns who have immature immunity $(7,8)$, the Expert Group On Prevention and Control of 2019$\mathrm{nCoV}$ Infection in the Perinatal Period has put forward several recommendations for the prevention and control of neonatal 2019-nCoV infection, which are based upon the historical literature of coronavirus infection and the latest 2019-nCoV infection data that could be acquired. These recommendations are in accordance with "The guidelines for the diagnosis and management plan for pneumonia with new coronavirus infection (trial version 4)" (1), "Technical guidelines for prevention and control of the new coronavirus infection in medical institutions", "The guidelines on the scope of use of common medical protection against new coronavirus infection" (trial), and the latest World Health Organization (WHO) principles for the treatment of 2019-nCoV infection $(9,10)$.

The Chinese Perinatal-neonatal 2019-nCoV Committee has undertaken a systematic review of all recent and previous peer-reviewed literature relating to the 2019$\mathrm{nCoV}$, SARS-CoV, and MERS-CoV infection in adults, children and infants, focusing on publications that delineate clinical epidemiological characteristics, risk factors, clinical findings, management, and outcome in the individuals infected by 2019-nCoV, SARS- CoV, or MERS-CoV.

Infants with 2019-nCoV infection are classified according to the presence or absence of apparent symptoms, which may be similar to those of adults. Their clinical manifestations may be asymptomatic, mild, or severe. Available data show that the incubation period is generally
3-7 days, with the shortest being 1 days, and the longest being 14 days $(7,9,10)$. Diagnosis of infection requires the detection of a highly homologous sequence with known 2019-nCoV in the upper respiratory tract (URT; nasopharyngeal and oropharyngeal), lower respiratory tract (LRT; sputum, endotracheal aspirate, or bronchoalveolar lavage), or serum $(1,10)$. It has been found that the nuclear acid of the 2019-nCoV virus can be detected in fecal samples of infected people, although the route of aerosol and digestive tract transmission is still unclear $(1,10)$.

\section{Neonatal clinical manifestations associated with 2019-nCoV infection $(7,8,11-13)$}

\section{Clinical findings}

Clinical findings, especially premature infants, are not specific. Therefore, it is necessary to closely monitor vital signs, respiratory symptoms and gastrointestinal symptoms.

Temperature instability: the temperature of an infected infant may be elevated, depressed, or normal.

Respiratory and cardiovascular symptoms: these symptoms may include tachypnea, grunting, nasal flaring, work of breathing (WOB), apnea, cough, or tachycardia.

Other findings: other findings may include poor feeding, lethargy, vomiting, diarrhea, and abdominal distension.

\section{Laboratory findings}

Laboratory examinations may be non-specific.

Complete blood count (CBC): an early exam may show normal or decreased leukocyte counts, or decreased lymphocyte counts.

Other findings: other findings may include mild thrombocytopenia, and elevated levels of creatine kinase, alkaline phosphatase, alanine aminotransferase, aspartate aminotransferase, and lactate dehydrogenase.

2019-nCoV can be detected in the upper respiratory tract (URT; nasopharyngeal and oropharyngeal), the lower respiratory tract (LRT; endotracheal aspirate, or bronchoalveolar lavage), the blood and the stool.

\section{Radiographic findings}

Chest imaging: radiography, or lung ultrasound is likely to show pneumonia.

Abdominal radiography: this may show the characteristic radiographic features of intestinal ileus. 


\section{Definition of suspected 2019-nCoV infection}

The definition for newborns suspected of 2019-nCoV infection are those newborns born to the mothers with a history of 2019-nCoV infection between 14 days before delivery and 28 days after delivery, or the newborns directly exposed to those infected with 2019-nCoV (including family members, caregivers, medical staff, and visitors). Suspected infants are under consideration regardless of whether they present symptoms.

\section{Definition of confirmed 2019-nCoV infection}

Diagnosis of 2019-nCoV infection can be confirmed if 1 of the following etiological criteria is met:

(I) Respiratory tract or blood specimens tested by real-time fluorescence polymerase chain reaction (RT-PCR) are positive for 2019-nCoV nucleic acid;

(II) Virus gene sequencing of the respiratory tract or blood specimens is highly homologous to that of the known 2019-nCoV specimens.

\section{Suspected asymptomatic neonates (Figure 1)}

\section{Laboratory tests}

Laboratory tests should include CBC, C-reactive protein (CPR), and 2019-nCoV detection by RT-PCR.

It is recommended that samples are collected from multiple sites, including two specimen types: the upper respiratory tract (URT; nasopharyngeal and oropharyngeal), the lower respiratory tract (LRT; endotracheal aspirate, or bronchoalveolar lavage), and the blood. Additional specimen types (e.g., stool, urine) may be collected and stored $(1,10)$.

\section{Medical management}

These neonates should be admitted to the quarantine ward. Tertiary prevention should be taken and close monitoring and supportive care are essential. Heart rate, respiration, oxygen saturation, temperature, blood pressure, blood glucose, and gastrointestinal symptoms should be continuously monitored. Feeding plan is based on whether mother is infected with 2019-nCoV. See details in "Breast Milk".

\section{Suspected symptomatic neonates (Figure 1)}

\section{Laboratory tests}

Laboratory tests should include CBC, CRP, 2019-nCoV detection by RT-PCR, and chest radiography or lung ultrasound. If necessary, tests of liver and kidney function, along with cardiac biomarkers and abdominal radiography, can also be conducted. Other tests should be considered to rule out other etiologies of infection (such as influenza viruses, respiratory syncytial virus, bacterium, etc.).

\section{Medical management}

These neonates should be admitted to the quarantine ward. Tertiary prevention should be taken. Feeding plan is based on whether mother is infected with 2019-nCoV.See details in "Breast Milk".

If patient results are negative for $2019-\mathrm{nCoV}$, they can be managed based on the etiology of infection.

\section{Diagnosed asymptomatic neonates (Figure 1)}

\section{Laboratory tests}

Laboratory tests should include CBC, CRP, 2019-nCoV detection by RT-PCR.

\section{Radiography}

Chest imaging or lung ultrasound is recommended.

\section{Medical management}

The neonates should be admitted to the quarantine ward. Tertiary prevention should be taken and close monitoring and supportive care are essential. Feeding plan is based on whether mother is infected with 2019-nCoV.See details in "Breast Milk".

\section{Diagnosed symptomatic neonates (Figure 1)}

\section{Laboratory tests}

Laboratory tests should include CBC and CRP, blood gas analysis, acid-base studies, serum electrolyte, liver and kidney function with cardiac biomarkers, and 2019-nCoV detection by RT-PCR. Other etiologies of infection (such 


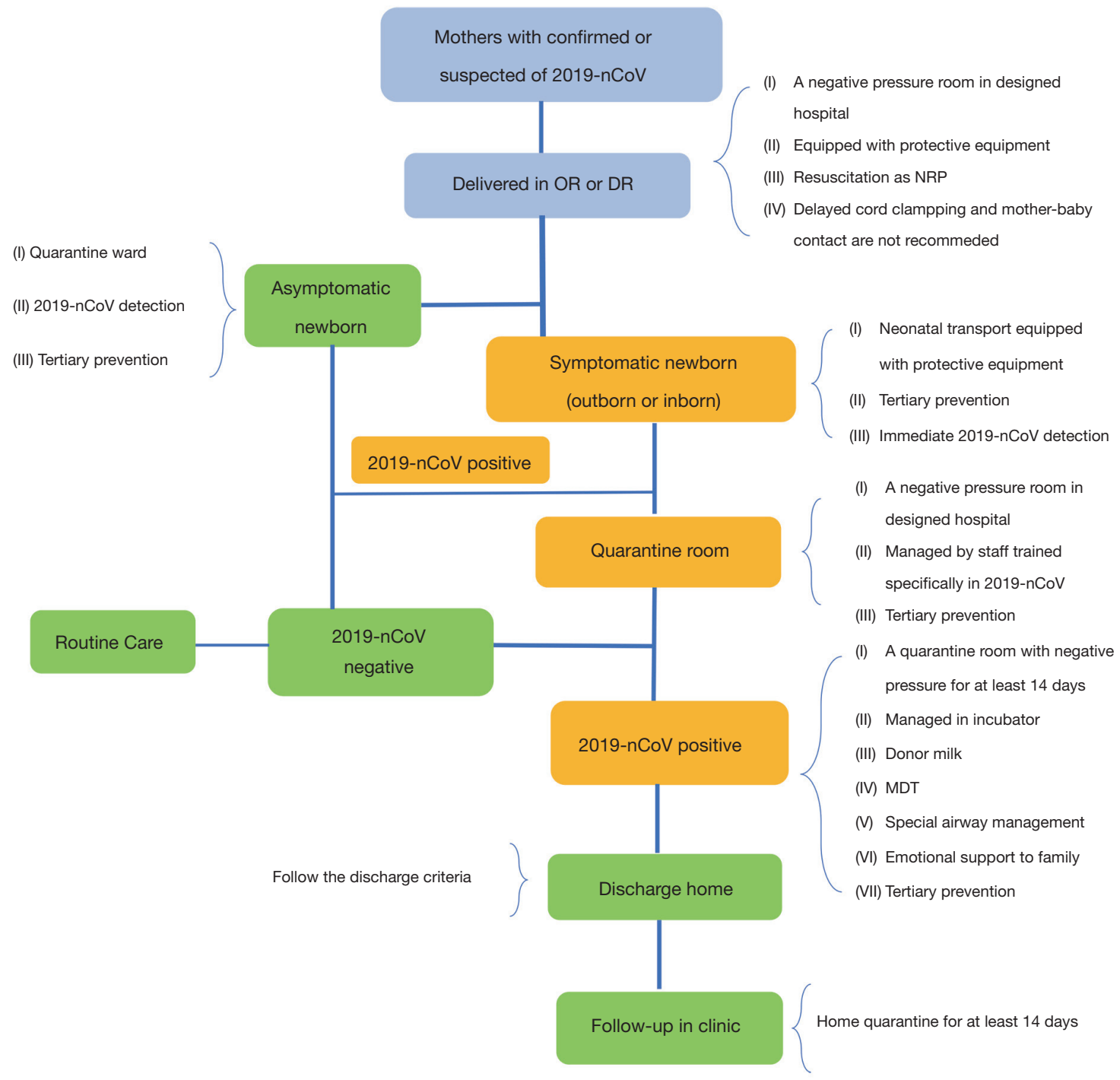

Figure 1 Flowsheet for the perinatal-neonatal management of suspected and confirmed 2019 novel coronavirus infection.

as influenza viruses, respiratory syncytial virus, bacterium, etc.) should be ruled out.

\section{Radiography}

Chest imaging (radiography, or lung ultrasound) and abdominal radiography can be performed if necessary.

\section{Medical management}

These neonates should be admitted to the quarantine ward. Tertiary prevention should be taken. Currently, the principle of treatment is supportive care and treatment of complications.

For infants with severe acute respiratory distress syndrome, high-dose pulmonary surfactant (PS), inhaled nitric oxide (iNO), and high-frequency oscillatory ventilation (HFOV) may be effective. For critically ill cases, continuous renal replacement therapy (CRRT) and extracorporeal membrane lung (ECMO) therapy are required.

A multi-disciplinary team (MDT) for the management of critical neonates with $2019-\mathrm{nCoV}$ infection is recommended. MDTs involve combinations of the following sub-specialties trained in 2019-nCoV prevention: obstetric, neonatal, and intensive care specialists, pulmonologists, radiologists, specialists of infection, specialist nurses, and 
social workers.

Currently, there exists no effective anti-coronavirus drug $(9,10,12,13)$. Inappropriate use of antibiotics, especially broad-spectrum antibiotics, should be avoided. If there is evidence of secondary bacterial infection, the relevant antibiotics should be used. There is no evidence supporting the effectiveness of gamma globulin, interferon, or hormone therapy.

\section{Discharge criteria (1)}

\section{Asymptomatic infection}

Upper airway specimens (nasopharyngeal and pharyngeal swabs) should be collected and tested every 2 days (with at least a 24-hour interval) until 2 consecutive results show negative for 2019-nCov. Stool may be collected and tested every 2 days (with at least a 24-hour interval) until two consecutive results show negative for 2019-nCoV (6).

\section{Mild infection}

The temperature of the patient should be normal for more than 3 days, symptoms should improve, and specimens collected from the upper airway secretion (nasopharyngeal and pharyngeal swabs) should show negative for 2019-nCov 2 consecutive times (with at least a 24-hour interval). Stool may be collected and tested every 2 days until 2 consecutive results show negative for 2019-nCoV (6).

\section{Severe infection}

The temperature should return to normal for more than 3 days, symptoms should improve, and pulmonary imaging should show inflammation disappearing. Specimens collected from the upper respiratory tract (nasopharyngeal and pharyngeal swabs) and lower respiratory tract (sputum) should show negative for 2019-nCov 2 consecutive times (with at least a 24-h interval). Stool may be collected and tested every 2 days until 2 consecutive results show negative for 2019-nCoV (6).

\section{Neonates born to mothers suspected for or diagnosed with 2019-nCoV infection (Figure 1)}

The newborns of pregnant women infected with SARS are mostly premature infants, and, based on the similarity between 2019-nCoV and SARS, intrapartum management can proceed accordingly. Thus, obstetrics and pediatrics must jointly evaluate the maternal and fetal conditions and choose the best time, mode, and place for delivery (14). For example, a special delivery room or operating room (a negative pressure room is recommended) can be prepared along with an isolation protection device, so as to minimize the damage to the mother and fetus.

Resuscitation of neonates should be carried out according to the Neonatal Resuscitation Program (NRP) 7th edition. Physicians must wear protective equipment (including hats, goggles, protective suits, gloves, N95 masks, etc.).

To reduce the risk of the vertical transmission of 2019$\mathrm{nCoV}$, delayed cord clamping (DCC) is not recommended. Mother-baby contact is also not recommended.

\section{Neonatal transport}

The transport ambulance should be equipped with special transfer incubators for newborns, transport ventilators along with other newborn intensive care unit (NICU) monitoring equipment, transport supplies, and first aid medicine. The cab and the car should be strictly sealed and isolated, and the car should be equipped with protective equipment, disinfectant solution, and rapid hand disinfectant. The ambulance should be disinfected before and after transfer. Also, any accompanying medical staff must wear protective equipment.

\section{Breast milk}

The possibility of the vertical transmission of 2019$\mathrm{nCoV}$ cannot be ruled out. Infants should not be fed with the breast milk from mothers with confirmed or suspected of 2019-nCoV. If the suspected or diagnosed mother and her breast milk test negative for 2019-nCoV, infants should be fed with breast milk. Donor milk can be considered for use after being screened for 2019-nCoV, because the virus may be excreted into the milk during the incubation period.

\section{Isolation}

The delivery room for those infected should be a special room (preferably a negative pressure room, since some SARS cases were detected in maternal ascites) (11). The accompanying medical staff must equip with protective equipment. 
There should be separate rooms for benign, suspected, and confirmed cases. Suspected and confirmed neonates should be treated in designated hospitals with effective isolation and protective equipment. Suspected newborns should be isolated and treated in a single room, and confirmed cases should be admitted into separate rooms from suspected ones.

The neonatal department should be subdivided into transitional, quarantine, and general wards. Before neonates enter the department, doctors should determine which ward they should be assigned to base on the results of triage. Suspected or confirmed neonates are suggested to be placed in an incubator. Usage of an open rescue table should be prohibited in order to avoid cross transmission of the virus. Diagnosis and treatment items (stethoscope, thermometer, etc.) and nursing facilities should be kept for their particular use. To enter and exit the quarantine ward, proper protocol for hand hygiene and the deployment of protective equipment should be strictly followed.

\section{Airway management}

For all operations that may produce aerosols (such as endotracheal intubation, non-invasive ventilation, cardiopulmonary resuscitation, manual ventilation before intubation, bronchoscopy, etc.) on suspected or diagnosed neonates, the following measures are necessary: (I) air isolation measures should be prepared; (II) medical masks should be worn and tightness tests conducted; (III) eye protection (such as goggles) should be applied; (IV) longsleeved protective suits and gloves should be worn to block body fluid; (V) operations should be carried out in a well-ventilated room; (VI) the number of people in the room should be minimized, with medical staff making the necessary operation clusters to reduce contact with patients as much as possible. As a final measure (VII), a head cover should be placed on the suspected neonates who are using auxiliary ventilation. A negative pressure suction tube can be placed in the head cover, with the outlet end of the ventilator connected with a filter and then connected to a negative pressure suction device; a closed loop sputum suction tube can be affixed to the sputum suction with a resuscitation bag for the artificial ventilation connected with the filter device.

\section{Disinfection}

The medical wastes produced by infants should be thrown in double-layer infectious medical waste bags, the disinfection effect of chlorine-containing solution should be applied for more than 10 minutes (the concentration of disinfectant should follow the requirements of disinfection of infectious disease foci), and then the waste should be disposed of according to infectious medical waste protocol. Patients' non-disposable medical fabrics should be collected by the bedside and disinfected with chlorine-containing solution for more than 10 minutes (the concentration of disinfectant should also comply with local recommendations), and then be disposed of according to protocols of infectious medical fabric. The final disinfection of the patient's room should involve hydrogen peroxide atomization or gasification, or chlorine-containing solution spray disinfection.

\section{Communication}

Thus far, there have been no cases of mother-tochild vertical transmission reported. If the newborn is asymptomatic after birth and the suspected mother tests negative for 2019-nCoV, the newborn can be roomed with the mother; if the maternal testing is positive for 2019$\mathrm{nCoV}$, maternal quarantine is required, the neonate needs to be isolated, and a 2019-nCoV test should be conducted. If the test is negative, the newborn can be routinely cared for by the guardian; however, if the test is positive, the case should be managed in accordance with the management of diagnosed newborns. If the infants show clinical symptoms of suspected 2019-nCoV, they need to be admitted into the neonatal department for further diagnosis and treatment.

Maternal separation may cause anxiety to parents and maternal depression (14), and thus psychological comfort should be offered, and the newborns should be fed with donor milk. All of the above situations should be communicated to and fully understood by parents. Social workers or supportive consultants can provide help to facilitate communication.

\section{Summary}

* The 2019-nCoV infection may cause harm to newborns who have immature immunity. Neonates with positive 2019-nCoV tests have been reported.

- Clinical manifestations of 2019-nCoV infection in neonates may be non-specific. Therefore, it is necessary to closely monitor and identify them. 
Neonates at great exposure to $2019-\mathrm{nCoV}$ should be isolated and tested for 2019-nCoV infection.

- Currently, no effective anti-coronavirus drug exists. Inappropriate use of antibiotics, especially broadspectrum antibiotics, should be avoided. Close monitoring and supportive care are a priority. MDTs for the management of critical neonates with 2019$\mathrm{nCoV}$ infection is recommended.

* The possibility of vertical transmission of 2019-nCoV cannot be ruled out. Thus, delayed cord clamping is not recommended and infants should not be fed with breast milk from mothers with confirmed 2019-nCoV.

* The neonatal department should be subdivided into transitional, quarantine, and general wards. The delivery room and neonatal transport for neonates suspected for or diagnosed with 2019-nCoV should be equipped with protective equipment, disinfectant solution, and rapid hand disinfectant.

* For all operations that may produce aerosols on suspected or confirmed infants, it is better to take airborne precautions.

- Physicians and nurses who take care of neonates suspected for or confirmed with 2019-nCoV should be equipped with protective equipment and receive psychological support.

* Family support should be offered in the case of maternal separation and depression. Social workers or supportive consultants can provide help to facilitate communication.

\section{Acknowledgments}

The authors would like to thank Bin Chen for revising the manuscript.

\section{Footnote}

Conflicts of Interest: The authors have no conflicts of interest to declare.

Ethical Statement: The authors are accountable for all aspects of the work in ensuring that questions related to the accuracy or integrity of any part of the work are appropriately investigated and resolved.

\section{References}

1. Diagnosis and management plan of pneumonia with new coronavirus infection (trial version 4). Beijing: National Health and Health Commission, 2020.

2. Zhu N, Zhang D, Wang W, et al. A novel coronavirus from patients with pneumonia in China, 2019. N Engl J Med 2020. [Epub ahead of print].

3. World Health Organization. Laboratory testing for 2019 novel coronavirus (2019-nCoV) in suspected human cases. (2020-01-14). Available online: https://www.who.int/docs/ default-source/coronaviruse/20200114-interim-laboratoryguidance version.pdf?sfvrsn=6967c39b_4\&download=true

4. World Health Organization. SARS (severe acute respiratory syndrome) (2019) [(EB/OL]). Available online: https://www.who.int/ith/diseases/sars/en/

5. Azhar EI, Hui DSC, Memish ZA, et al. The middle east respiratory syndrome (MERS). Infect Dis Clin North Am 2019;33:891-905.

6. Zhang H, Kang Z, Gong H, et al. The digestive system is a potential route of 2019-nCov infection: a bioinformatics analysis based on single-cell transcriptomes. bioRxiv, 2020, 2020.01.30.927806.

7. Fang F, Luo XP. Facing the pandemic of 2019 novel coronavirus infections: the pediatric perspectives. Chin J Pediatr 2020;58:81-5.

8. Li AM, Ng PC. Severe acute respiratory syndrome (SARS) in neonates and children. Arch Dis Child Fetal Neonatal Ed 2005;90:F461-5.

9. Zhang N, Wang L, Deng X, et al. Recent advances in the detection of respiratory virus infection in humans. J Med Virol 2020. [Epub ahead of print].

10. World Health OrganizationWHO. Interim clinical guidance for management of patients with confirmed 2019 novel coronavirus (2019-nCoV) Infection. Available online: https://www.cdc.gov/coronavirus/2019-ncov/hcp/ clinical-guidance-management-patients.html

11. Shek CC, Ng PC, Fung GP, et al. Infants born to mothers with severe acute respiratory syndrome. Pediatrics 2003;112:e254.

12. Huang C, Wang Y, Li X, et al. Clinical features of patients infected with 2019 novel coronavirus in Wuhan, China.

Lancet 2020. DOI: https://doi.org/10.1016/S01406736(20)30183-5.

13. Cai JH, Wang XS, Ge YL, et al. First case of 2019 novel coronavirus infection in children in Shanghai. Chin J Pediatr 2020;58:E002.

14. Maternal and Fetal Experts Committee, Chinese Physician Society of Obstetrics and Gynecology, Chinese Medical Doctor Association, Obstetric Subgroup, Society of Obstetrics and Gynecology, Chinese Medical 
Association, Society of Perinatal Medicine, Chinese Medical Association, Editorial Board of Chinese Journal of Perinatal Medicine. Proposed management of 2019-novel coronavirus infection during pregnancy and puerperium. Chin J Perinat Med 2020;23:73-9.

Cite this article as: Wang L, Shi Y, Xiao T, Fu J, Feng X, Mu D, Feng Q, Hei M, Hu X, Li Z, Lu G, Tang Z, Wang Y, Wang C, Xia S, Xu J, Yang Y, Yang J, Zeng M, Zheng J, Zhou W, Zhou X, Zhou X, Du L, Lee SK, Zhou W; on behalf of the Working Committee on Perinatal and Neonatal Management for the Prevention and Control of the 2019 Novel Coronavirus Infection. Chinese expert consensus on the perinatal and neonatal management for the prevention and control of the 2019 novel coronavirus infection (First edition). Ann Transl Med 2020;8(3):47. doi: 10.21037/atm.2020.02.20 